\title{
Auxílio multicritério à decisão aplicado ao planejamento e gestão na indústria de petróleo e gás
}

\author{
Roberta Braga Neves, Valdecy Pereira ${ }^{\mathrm{b}}$, Helder Gomes Costa ${ }^{\mathrm{c} *}$ \\ arobertaneves@producao.uff.br, UFF, Brasil \\ bvaldecy.pereira@gmail.com, UFF, Brasil \\ c*hgc@vm.uff.br, UFF, Brasil
}

\begin{abstract}
Resumo
A utilização de métodos multicritério no planejamento e gestão da indústria de petróleo e gás tem sido estudada por alguns autores. No entanto, esses estudos encontram-se distribuídos de forma dispersa na literatura, o que torna difícil uma visão panorâmica a respeito do tema. Objetivando fornecer uma visão sistemática sobre esse assunto, este artigo apresenta um estudo sobre as publicações no âmbito da aplicação de métodos multicritério ao planejamento e gestão da indústria de petróleo e gás. Foram analisados 48 artigos, selecionados através de uma pesquisa sistemática efetuada nas bases Scopus e ISI (Web of Knowledge). Dentre os resultados do estudo, destaca-se que o método multicritério AHP (Analytic Hierarchy Process) foi o mais utilizado. Outro resultado de destaque é que a maioria das aplicações estavam relacionados a: petróleo, atividades de midstream e instalações onshore.
\end{abstract}

Palavras-chave

Multicritério. Planejamento. Gestão. Petróleo. Gás.

\section{Introdução}

Conforme reportado em Ratnayake e Markeset (2010), a indústria de petróleo e gás (P\&G) atua em um mercado cada vez mais complexo devido à crescente concorrência global, variações dos preços, rigorosas exigências de SMS (Saúde, Meio Ambiente e Segurança), crescimento de fontes alternativas de energia e pressões dos stakeholders em relação a interesses ambientais, sociais, econômicos e políticos.

Nesse âmbito, a busca pelo padrão de excelência em tecnologia, planejamento e gestão, aliada ao investimento no aumento da capacidade para atender as demandas são importantes para o desenvolvimento da indústria de $P \& G$ e induzem decisões e análises que abrangem múltiplos critérios, além de diferentes grupos de stakeholders (indústria, fornecedores de bens e serviços, governos, cidadãos, grupos ambientalistas, organismos e instituições internacionais e ONGs, dentre outros).

Nesse contexto, a adoção de métodos e ferramentas de apoio à decisão que sejam capazes de avaliar problemas à luz de uma perspectiva múltipla - tais como os métodos de auxílio multicritério à decisão (AMD) - tem ocorrido. Nele, surge a seguinte questão: como tem evoluído a aplicação de métodos de AMD no contexto da indústria de petróleo e gás, ao longo dos anos?

0 presente trabalho objetiva responder a essa questão, efetuando uma revisão de literatura nos periódicos das bases Scopus e ISI (Web of Knowledge), acessados através do portal Capes, integrando os seguintes temas: petróleo e gás, planejamento e gestão e auxílio multicritério à decisão.

A importância da indústria de $P \& G$ para a sociedade global justifica a realização da pesquisa, visto que petróleo e gás são as fontes energéticas mais utilizadas atualmente. Assim sendo, os resultados do trabalho podem ser úteis como fonte de pesquisa para pesquisadores e decisores atuantes na indústria.

\section{Metodologia}

Os passos metodológicos da pesquisa foram inspirados nos trabalhos de Costa (2010) e Rodriguez, Costa e Do Carmo (2013), tendo pesquisadas duas bases científicas disponíveis para acesso pelo portal 
de periódicos Capes, são elas: Scopus e ISI (Web of Knowledge). Essas bases foram selecionadas devido à sua abrangência e ao seu reconhecimento como fontes de difusão do conhecimento científico. A pesquisa foi concluída no mês de junho de 2012, utilizando a seguinte frase de pesquisa: (oil OR gas OR petroleum) AND (planning OR management OR project OR design), busca no título e/ou resumo, considerando apenas artigos. Com essa frase de pesquisa, 92.088 registros foram encontrados no Scopus e 3.969 na base ISI. Em cada base, filtros foram aplicados a esses conjuntos de registros, objetivando identificar artigos que contemplassem o uso de métodos multicritério no contexto da indústria de $\mathrm{P} \& \mathrm{G}$.

A aplicação das palavras-chave e do filtro de pesquisa possibilitou a redução do conjunto de registros aos potencialmente aderentes ao tema da pesquisa. A análise do resumo desses registros resultou em um conjunto com 48 artigos validados como aderentes para a inclusão na revisão, sendo 47 da base Scopus e 5 da base ISI (4 eram comuns às duas bases).

É importante registrar que a frase de pesquisa e os filtros foram utilizados como "suporte à decisão" a fim de fornecer uma base inicial de artigos, para posterior refinamento. Essa é uma estratégia usual em pesquisas que envolvem uma revisão sistematizada da literatura, utilizada para eliminar ruídos associados a homonímia. Por exemplo: o termo ANP, além de ser a sigla de um método multicritério, também é sigla da Agência Nacional de Petróleo, fato que justifica a necessidade de se efetuar esse refinamento a partir da base inicial de artigos "potencialmente aderentes" à pesquisa.

0 Quadro 1 sintetiza os principais resultados oriundos dessa busca. A segunda coluna desse quadro apresenta na primeira linha a frase inicial de pesquisa, ao passo que as demais linhas dessa coluna ilustram os filtros associados ao termo multicritério aplicados aos resultados encontrados com a frase de pesquisa inicial. As duas últimas colunas do Quadro 1 ilustram, para as duas bases pesquisadas, os resultados encontrados pela aplicação da frase de pesquisa inicial e, também, pela aplicação de cada filtro. 0 Quadro 2 sintetiza as características gerais dos 48 artigos selecionados.

A seguir apresenta-se a análise dos dados desse quadro e, também, uma síntese de cada um dos artigos listados na mesma.

\section{Estatística descritiva dos artigos pesquisados.}

Os artigos selecionados foram analisados, do ponto de vista da estatística descritiva, de acordo com as seguintes classificações: (1) análise por método multicritério utilizado; (2) análise por ano de

Quadro 1. Filtro por método multicritérios.

\begin{tabular}{|c|c|c|c|}
\hline & \multirow{2}{*}{ Temos da pesquisa } & \multicolumn{2}{|c|}{ Resultados } \\
\hline & & Scopus & ISI \\
\hline $\begin{array}{l}\text { Frase inicial de pesquisa, restrita a busca } \\
\text { apenas por artigos }\end{array}$ & $\begin{array}{l}\text { (Oil OR Gas OR Petroleum) AND (Planning OR Management OR Project OR } \\
\text { Design) }\end{array}$ & 92.088 & 3.969 \\
\hline \multirow{23}{*}{ Filtros associados ao termo multicritério } & Aggregated Indices Randomization Method OR AIRM & 0 & 0 \\
\hline & Analytic Hierarchy Process OR AHP & 189 & 3 \\
\hline & Analytic Network Process OR ANP & 83 & 0 \\
\hline & Borda & & 0 \\
\hline & Condorcet & 0 & 0 \\
\hline & Disaggregation - Aggregation Approaches OR UTA OR UTAll OR UTADIS & 0 & 0 \\
\hline & Dominance-based Rough Set Approach OR DRSA & 0 & 0 \\
\hline & Elimination et Choix Traduisant la Realité $O R$ ELECTRE & 19 & 0 \\
\hline & Evidential Reasoning Approach & 4 & 0 \\
\hline & Geometrical Analysis for Interactive Aid $O R$ GAlA & 0 & 0 \\
\hline & Grey Relational Analysis OR GRA & 0 & 0 \\
\hline & $\begin{array}{l}\text { Measuring Attractiveness by a Categorical Based Evaluation Technique } O R \\
\text { MACBETH }\end{array}$ & 30 & 0 \\
\hline & Multi-attribute Global Inference of Quality OR MAGIQ & 0 & 0 \\
\hline & Multi-attribute Utility Theory $O R$ MAUT & 1 & 1 \\
\hline & Multi-attribute Value Theory OR MAVT & 0 & 0 \\
\hline & New Approach to Appraisal & 0 & 0 \\
\hline & Potentially all pairwise rankings of all possible alternatives $O R$ PAPRIKA & 14 & 0 \\
\hline & $\begin{array}{l}\text { Preference Ranking Organization Method for Enrichment of Evaluations } O R \\
\text { PROMETHEE }\end{array}$ & 21 & 0 \\
\hline & THOR & 0 & 0 \\
\hline & TODIM & 1 & 0 \\
\hline & TOPSIS & 1 & 1 \\
\hline & Weighted Product Model OR WPM & 0 & 0 \\
\hline & Weighted Sum Model OR WSM & 0 & 0 \\
\hline
\end{tabular}


Quadro 2. Lista de artigos selecionados para análise.

\begin{tabular}{|c|c|c|c|c|}
\hline Autor (ano de publicação) & Método multicritério & Fonte energética & Segmento da indústria & Situação geográfica \\
\hline Alidi (1996) & AHP e Programação por Metas & Petróleo/Gás & Midstream & Onshore \\
\hline Dey, Tabucanon e Ogunlana (1996) & AHP e Programação por Metas & Petróleo & Midstream & Onshore \\
\hline Xiao et al. (1998) & AHP e Programação Linear Multiobjetivo & Petróleo & Upstream & Onshore \\
\hline Dey e Gupta (1999) & AHP & Petróleo/Gás & Midstream & Onshore \\
\hline Dey (2001a) & AHP & Petróleo/Gás & Midstream & Onshore/Offshore \\
\hline Dey (2001b) & AHP & Petróleo/Gás & Midstream & Onshore/Offshore \\
\hline Dey (2002) & AHP e Análise de Árvore de Decisão & Petróleo/Gás & Midstream & Onshore/Offshore \\
\hline Dey (2003) & AHP & Petróleo & Midstream & Onshore \\
\hline Sadiq et al. (2003) & $\mathrm{AHP}$ & Petróleo & Upstream & Onshore \\
\hline Sii e Wang (2003) & Evidential reasoning, AHP e Delphi & Petróleo/Gás & Upstream & Offshore \\
\hline Dey (2004) & AHP & Petróleo/Gás & Midstream & Onshore \\
\hline Sadiq et al. (2004) & AHP e Lógica Fuzzy & Petróleo & Upstream & Offshore \\
\hline Dey e Mukherjee (2005) & AHP e Análise de Árvore de Decisão & Petróleo & Midstream & Onshore \\
\hline Hui e Deli (2005) & AHP & Petróleo/Gás & Upstream & Onshore \\
\hline Jiang et al. (2005) & $\mathrm{AHP}$ & Gás & Midstream & Onshore \\
\hline Li e Zhang (2005) & AHP e Topsis & Gás & Midstream & Onshore/Offshore \\
\hline Liu (2005) & AHP e Programação Linear Multiobjetivo & Petróleo & Não declarado & Onshore/Offshore \\
\hline Bertolini e Bevilacqua (2006) & AHP e Programação por Metas & Petróleo & Midstream & Onshore \\
\hline Dey (2006) & $\mathrm{AHP}$ & Petróleo & Não declarado & Onshore \\
\hline Tesfamariam e Sadiq (2006) & AHP & Petróleo/Gás & Upstream & Offshore \\
\hline Yang et al. (2006) & AHP e lógica Fuzzy & Petróleo & Upstream & Onshore/Offshore \\
\hline Balasubramaniam, Boyle e Voulvoulis (2007) & ELECTRE 111 & Petróleo & Midstream & Offshore \\
\hline Massara et al. (2008) & AHP & Gás & Midstream & Onshore \\
\hline Sharma e Gandhi (2008) & AHP & Petróleo & Midstream & Onshore \\
\hline Xie et al. (2008) & Evidential Reasoning & Petróleo & Midstream & Offshore \\
\hline Araujo e Almeida (2009) & PROMETHEE 11 & Petróleo/Gás & Não declarado & Não declarado \\
\hline Brito e Almeida (2009) & MAUT & Gás & Downstream & Onshore \\
\hline Celik e Topcu (2009) & ANP & Petróleo & Upstream & Offshore \\
\hline Gomes, Rangel e Maranhão (2009) & TODIM & Gás & Midstream & Onshore \\
\hline Jafari, Nejadi e Abiri (2009) & AHP & Gás & Downstream & Onshore \\
\hline Montazer, Saremi e Ramezani (2009) & ELECTRE 111 & Petróleo/Gás & Não declarado & Não declarado \\
\hline Wang et al. (2009) & ANP & Petróleo/Gás & Midstream & Onshore/Offshore \\
\hline Yu, Tian e Xie (2009) & $\mathrm{AHP}$ & Gás & Downstream & Onshore \\
\hline Amiri (2010) & AHP e TOPSIS & Petróleo & Upstream & Não declarado \\
\hline Brito, de Almeida e Mota (2010) & ELECTRE TRI & Gás & Midstream & Onshore \\
\hline Dey (2010) & AHP & Petróleo & Midstream & Onshore \\
\hline Jozi e lraokhahi (2010) & AHP & Gás & Midstream & Onshore \\
\hline Massara e Udaeta (2010) & AHP & Gás & Downstream & Onshore/Offshore \\
\hline Ratnayake e Markeset (2010) & AHP & Petróleo/Gás & Upstream & Offshore \\
\hline Shafiq e Silvianita (2010) & AHP & Petróleo/Gás & Midstream & Onshore/Offshore \\
\hline Song, Tang e Jiang (2010) & AHP e Entropy Weight Method & Petróleo & Midstream & Onshore \\
\hline Chen et al. (2011) & AHP & Petróleo & Upstream & Offshore \\
\hline Dawotola, Van Gelder e Vrijling (2011) & AHP & Petróleo/Gás & Midstream & Onshore/Offshore \\
\hline Tan et al. (2011) & AHP & Petróleo & Midstream & Onshore \\
\hline Wang et al. (2011) & AHP & Petróleo & Midstream & Onshore/Offshore \\
\hline Yang, Khan e Sadiq (2011) & AHP e lógica Fuzzy & Petróleo/Gás & Upstream & Offshore \\
\hline Yildirim e Yomralioglu (2011) & AHP & Gás & Midstream & Onshore/Offshore \\
\hline Zhang, Gao e Liu (2012) & AHP e Lógica Fuzzy & Petróleo/Gás & Upstream & Onshore/Offshore \\
\hline
\end{tabular}

publicação; (3) análise por fonte energética; (4) análise por segmento da indústria de P\&G; e (5) análise por situação geográfica.

Como observado Figura 1, o AHP (Analytic Hierarchy Process) é o método multicritério mais utilizado para planejamento e gestão na indústria de P\&G, representando 60,94\% das ocorrências, visto que alguns artigos apresentaram mais de um método. Essa predominância de aplicações do AHP no âmbito do apoio multicritério à decisão também foi observada em outros trabalhos como em: $\mathrm{Ho}, \mathrm{Xu}$ e Dey (2010), Wu e Barnes (2011) e Méxas, Quelhas e Costa (2012).

A Figura 2 apresenta o total de publicações por ano. Nota-se que os primeiros artigos foram publicados em 1996. Também pode ser observado que de 1997 a 2000 não houve qualquer ocorrência. E que 2009 e 2010 foram os anos que apresentaram o maior número de publicações (8 artigos), seguido pelo ano de 2011 (6 artigos).

A Tabela 1 mostra que, dos artigos que declararam o tipo de fonte energética estudado, 41,67\% referiam-se a petróleo, 35,42 \% a petróleo/gás e $22,92 \%$ a gás.

Conforme a Agência Nacional de Petróleo, Gás Natural e Bicombustíveis - ANP (2010), as atividades na indústria de $P \& G$ são classificadas em upstream, midstream e downstream. 0 termo upstream refere-se a todas as atividades relacionadas a exploração e produção; midstream está relacionado ao refino, 
processamento, transporte e armazenamento; já downstream compreende as atividades de distribuição e revenda do petróleo e gás.

A Figura 3 ilustra a distribuição dos artigos por segmento da cadeia. A análise desse gráfico permite observar que nenhum dos artigos selecionados abordou atividades relacionadas à distribuição e revenda de petróleo e gás. No entanto, 56,25\% dos trabalhos que declararam o segmento apresentam estudo com midstream; 27,08\% com upstream; e 8,33\% com downstream. Vale salientar que 8,33\% dos artigos não puderam ser identificados.

As instalações de P\&G são classificadas, de acordo com seu posicionamento geográfico, em onshore e offshore. Segundo a ANP (AGÊNCIA..., 2010), onshore refere-se às instalações localizadas em terra, já o termo offshore está relacionado com as instalações

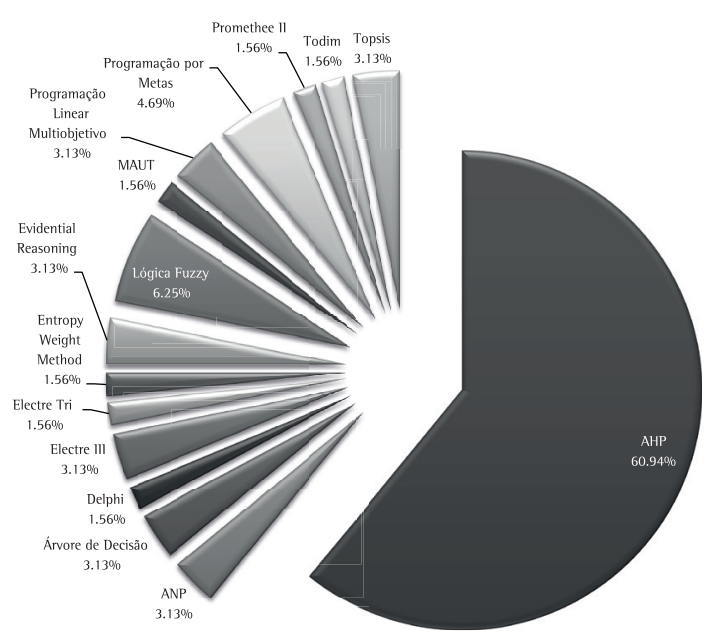

Figura 1. Análise por método multicritério.

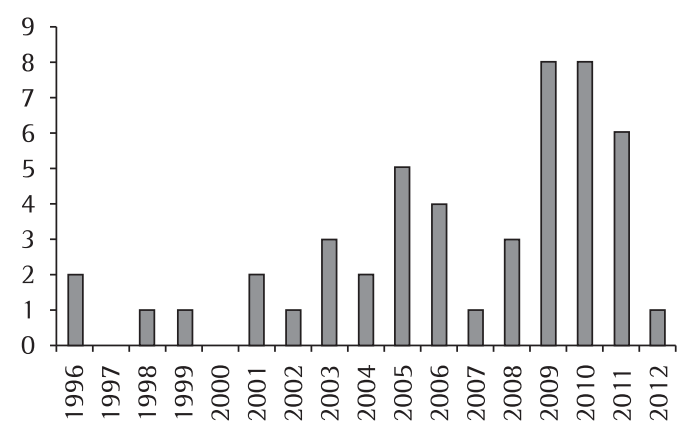

Figura 2. Análise por ano de publicação.

Tabela 1. Análise por tipo de fonte energética.

\begin{tabular}{cc}
\hline Fonte Energética & Percentual (\%) \\
\hline Petróleo/Gás & 35,42 \\
Petróleo & 41,67 \\
Gás & 22,92 \\
\hline
\end{tabular}

em alto-mar. Em 6,25\% dos artigos, não se identificou caracterização ou especificação quanto à localização onshore ou offshore. A Figura 4 mostra que 18,75\% dos artigos que especificaram a situação geográfica da instalação referiam-se a instalações offshore, ao passo que 47,92\% tratavam de localizações onshore e $27,08 \%$ referiam-se a ambas: onshore e offshore.

\section{Análise dos artigos}

A seguir é apresentada uma breve síntese dos artigos selecionados. Objetivando fornecer uma visão da evolução dos estudos sobre o tema pesquisado, a síntese dos artigos é apresentada em ordem cronológica.

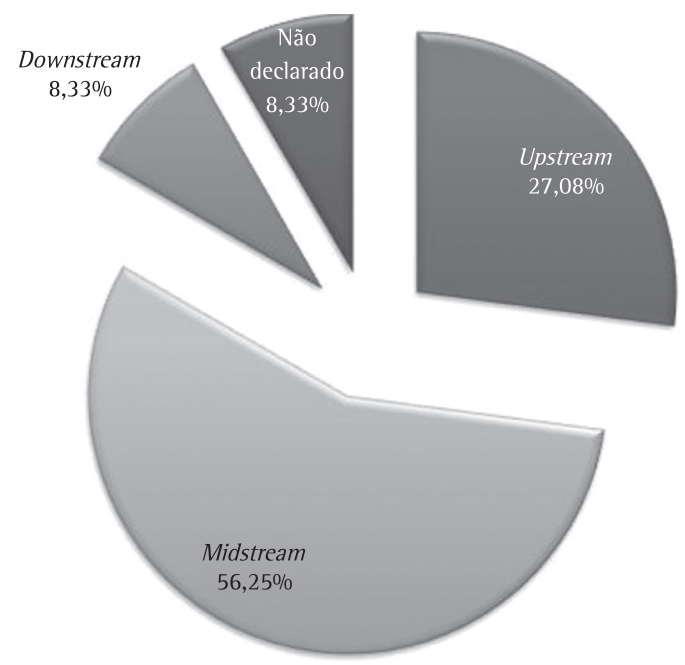

Figura 3. Análise por tipo de segmento de produção da cadeia de $\mathrm{P} \& \mathrm{G}$.

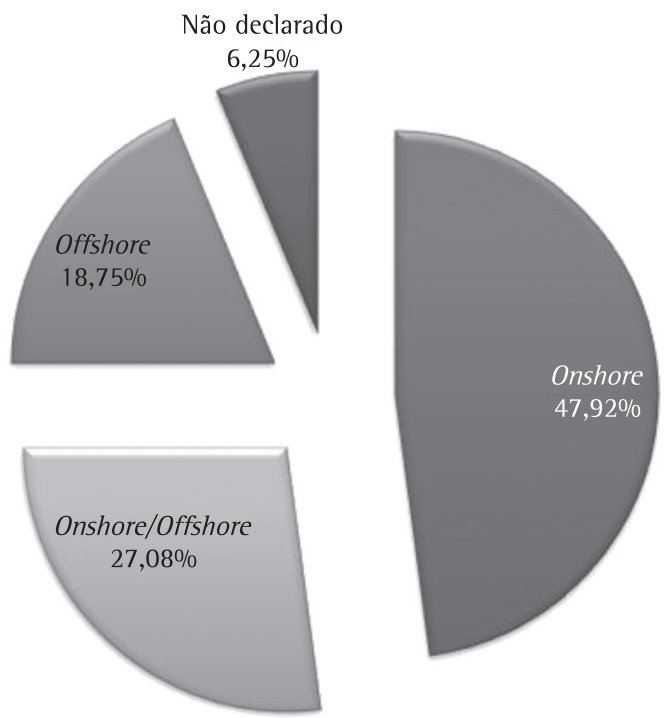

Figura 4. Análise por situação geográfica das instalações. 


\section{- Artigos publicados em 1996}

Alidi (1996) propõe, em seu estudo, um modelo baseado em programação por metas para auxiliar na gestão de resíduos perigosos gerados pela indústria petroquímica. $0 \mathrm{AHP}$, um método de apoio na tomada de decisão, é utilizado no modelo para priorizar as metas conflitantes encontradas geralmente ao abordar problemas dessa natureza. A aplicação do modelo foi ilustrada através de um exemplo numérico, usando dados hipotéticos, mas representativo para a tomada de decisão.

Dey, Tabucanon e Ogunlana (1996) abordam uma metodologia para o controle do projeto de um oleoduto na Índia através da análise de riscos, de reserva de recursos para contingências e de um modelo de planejamento hierárquico. A análise de risco é realizada através do método $\mathrm{AHP}$, devido à natureza subjetiva dos riscos nos projetos de construção. Os resultados dessa análise são utilizados para determinar a lógica de contingência com a aplicação da teoria de probabilidade. A programação por metas, uma técnica de decisão multicritério, é proposta para a formulação do modelo devido à sua flexibilidade e à sua estrutura de prioridades. 0 modelo possui três níveis hierárquicos: projeto, pacote de trabalho e atividade. A programação por metas é aplicada separadamente em cada nível. A eficácia no controle do projeto é demonstrada pelos autores.

\section{- Artigos publicados em 1998}

Xiao et al. (1998) propuseram um modelo de programação linear multiobjetivo no sistema de recuperação de campos petrolíferos. Um algoritmo modificado de pontos interiores usando conceitos de algoritmo Kamarkar e do AHP foi construído e os autores afirmam que o algoritmo é provavelmente mais eficiente do que algoritmos tradicionais para o processo de tomada de decisão no sistema de recuperação de campos petrolíferos através de um exemplo numérico.

\section{- Artigos publicados em 1999}

Dey e Gupta (1999) desenvolveram um sistema de apoio à decisão para a seleção de rotas de gasodutos que utiliza o método AHP - o sistema é demonstrado através de um estudo de caso de seleção rotas do gasoduto na índia.

\section{- Artigos publicados em 2001}

Dey (2001a) propôs uma metodologia de seleção de projetos de oleodutos de petróleo utilizando o método AHP e integrando os critérios de: análise de mercado (determinando exigências de lucratividade, oferta e demanda), análise técnica (identificando opções tecnológicas e alternativas de rotas de oleodutos), análise econômica e financeira (identificando as opções de menor custo), avaliação de impacto ambiental (abordando questões como a sugestão de locais alternativos, rotas, tecnologias e/ou metodologia de implementação). A metodologia foi demonstrada através de um estudo de caso de um projeto de oleoduto de petróleo na índia.

Dey (2001b) desenvolveu um modelo baseado em risco para a inspeção dutos de petróleo. 0 modelo utiliza o método AHP para identificar os fatores que influenciam a falha em segmentos específicos dos dutos e analisa os seus efeitos, determinando a probabilidade de ocorrência de fatores de risco. 0 modelo não elimina totalmente a subjetividade, mas é um aperfeiçoamento em relação ao método de inspeção tradicional existente.

\section{- Artigos publicados em 2002}

Dey (2002) demonstra uma abordagem quantitativa para gestão de risco de projetos de construção através do AHP e análise de árvore de decisão. Os pacotes de trabalho com risco são identificados com o auxílio dos stakeholders. Os fatores de risco também são identificados, seus efeitos são quantificados através da determinação de probabilidade (usando o AHP). Várias respostas alternativas são geradas, listando as implicações de custo de mitigar os riscos quantificados. Os valores monetários esperados são derivados para cada alternativa em uma estrutura de árvore de decisão, e análise de probabilidade posterior ajuda a tomar a decisão na gestão de riscos. A metodologia é explicada usando um estudo de caso de um projeto do duto de petróleo na índia.

\section{- Artigos publicados em 2003}

Dey (2003) propõe um modelo baseado no risco e que reduz o tempo gasto na inspeção de oleodutos. Esse modelo utiliza o método AHP para identificar os fatores que geram falhas em cada segmento do duto e para analisar seus efeitos, através da probabilidade dos fatores de risco. A gravidade da falha é determinada pela análise das consequências. A partir disso, o efeito de uma falha gerada por cada fator de risco pode ser estabelecido em termos de custo e o efeito cumulativo de falha é determinado por análise de probabilidade. A técnica não elimina totalmente a subjetividade, mas apresenta uma melhoria nos métodos de controle já existentes.

Sadiq et al. (2003) apresentam uma metodologia para orientar a tomada de decisão na seleção e avaliação de fluidos de perfuração utilizando o método AHP. A composição das lamas de perfuração é baseada em uma mistura de argilas e aditivos em um fluido base. Existem três categorias de fluido base - água, óleo e sintéticas. Os fluidos à base de água (WBFs) são relativamente benignos ao meio ambiente, mas o desempenho da perfuração é melhor com fluidos à base de óleo (OBFs). A indústria de petróleo e gás desenvolveu fluidos de base sintética (SBFs) que proporcionam um desempenho comparável aos OBFs, mas com menor efeito sobre a saúde ambiental e 
ocupacional. No estudo, uma comparação de fluidos de perfuração é feita considerando vários aspectos envolvidos no ciclo de vida dos fluidos de perfuração. Uma estrutura de quatro níveis hierárquicos foi desenvolvida para determinar o desempenho final das alternativas e os fluidos de base sintética (SBFs) foram apontados como a melhor opção.

Sii e Wang (2003) desenvolveram uma metodologia baseada em evidential reasoning (ER) para avaliação de projetos de produtos de engenharia offshore nas fases iniciais. 0 modelo possui como critérios o custo, a segurança e o desempenho técnico. Outros critérios podem ser incorporados ao modelo para ajudar os tomadores de decisão na escolha do projeto que melhor satisfaça as exigências dos clientes. Os autores também demostram que a metodologia também pode incluir outras técnicas de tomada de decisão como o AHP e o método Delphi. Um exemplo ilustrativo é usado para demonstrar a aplicação da metodologia.

\section{- Artigos publicados em 2004}

Dey (2004) expandiu seu modelo baseado no risco e que reduz o tempo gasto na inspeção de oleodutos. Esse novo modelo também utiliza o método AHP para identificar os fatores que geram falhas em cada segmento do duto e para analisar seus efeitos através da probabilidade dos fatores de risco. Porém, o modelo também otimiza o custo das operações dos dutos e ajuda a desenvolver uma política de manutenção para todo o sistema dutoviário, sugerindo um design e uma filosofia operacional para os novos dutos.

Sadiq et al. (2004) apresentam um sistema de apoio à decisão para a seleção da melhor opção de descarte de resíduos de perfuração offshore de petróleo. 0 sistema utiliza lógica Fuzzy e o método AHP para definir a melhor opção.

\section{- Artigos publicados em 2005}

Dey e Mukherjee (2005) apresentaram uma metodologia para o gerenciamento de riscos em projetos. Os fatores de risco são identificados, seus efeitos são analisados e respostas alternativas são geradas considerando os custos para mitigação dos riscos identificados. Uma estrutura de tomada de decisão é então formulada utilizando o método AHP ea análise de árvore de decisão. Valores monetários esperados são gerados para cada alternativa. As respostas que apresentam menor custo são selecionadas. Toda a metodologia foi explicada através de um estudo de caso de um projeto de oleoduto na Índia.

Hui e Deli (2005) desenvolveram um método de triagem para as principais técnicas de perfuração de poços de petróleo e gás que consideram a segurança como a base. 0 método AHP é utilizado e um exemplo em um campo de perfuração chinês foi abordado.

Jiang et al. (2005) utilizaram o método AHP para analisar o melhor projeto de construção de gasodutos em áreas de rios, tomando como exemplo a seleção de projetos de gasodutos para a travessia do rio Tang, na China.

Li e Zhang (2005) utilizaram o método AHP e o método TOPSIS para classificar os 4 projetos de transporte de gás no campo de gás natural de Luodai, na China. Com uma análise abrangente dos aspectos técnicos e econômicos, dentro outros, a classificação dos projetos serviu como base para a escolha do melhor projeto.

Liu (2005) abordou problema de seleção de fornecedores de petróleo bruto e planejamento de aquisições em uma cadeia de abastecimento múltipla. Um modelo com múltiplos objetivos foi desenvolvido, para minimizar os custos totais, atender à qualidade, à entrega e ao tempo de ciclo. 0 modelo foi resolvido através de uma programação linear multiobjetivo associada ao método AHP. 0 autor afirma que a abordagem proposta para seleção de fornecedores e planejamento de aquisições é eficiente e aplicável.

\section{- Artigos publicados em 2006}

Bertolini e Bevilacqua (2006) utilizam a programação por metas combinada com o método AHP para definir as melhores estratégias na manutenção de bombas centrífugas em uma refinaria de petróleo italiana. 0 modelo, aplicado em duas fases, permite ao analista atribuir diferentes prioridades aos objetivos considerados. A primeira parte da análise (método AHP) define uma estrutura hierárquica de três níveis: o nível superior representa o objetivo da análise (no caso, a definição da política de manutenção), o segundo nível é relativo aos critérios relevantes utilizados (severidade, ocorrência e detecção), o terceiro define as alternativas possíveis. A segunda fase (programação por metas) identifica o melhor conjunto de políticas de manutenção para os tipos de falha ocorridos no equipamento considerado. Os autores apresentam dados e resultados do caso analisado a fim de mostrar as características e o desempenho da abordagem.

Dey (2006) propôs um sistema de apoio à decisão que analisa os projetos em geral com relação ao mercado, a aspectos técnicos e ao impacto social e ambiental em uma estrutura integrada utilizando o AHP. 0 sistema reduz a duração da avaliação e seleção de projetos, mas também ajuda a selecionar projetos ideais para a organização visando um desenvolvimento sustentável. Toda a metodologia foi aplicada a um projeto de oleoduto de petróleo na Índia.

Tesfamariam e Sadiq (2006) afirmam que gestão de risco ambiental é parte integrante das análises de risco. A seleção de diferentes alternativas mitigadoras ou preventivas envolve, muitas vezes, critérios concorrentes e conflitantes, o que requer a utilização de métodos multicritérios para a tomada de decisão. 0 método AHP, empregado na pesquisa, considera preferências 
pessoais e subjetivas na análise de desempenho, o que introduz incerteza por imprecisão. No estudo, a incerteza por imprecisão é considerada, utilizando técnicas Fuzzy. 0 AHP tradicional é modificado para AHP Fuzzy ao empregar operações aritméticas difusas. Os conceitos de atitude ao risco (tolerância) e de confiança do decisor nas estimativas de comparações paritárias são discutidos. A metodologia proposta é ilustrada em um exemplo hipotético e sua eficácia é demonstrada através de uma aplicação na seleção de fluido/lama de perfuração nas operações offshore de petróleo e gás.

Yang et al. (2006) desenvolveram um método de avaliação para fornecer suporte à tomada de decisões para investimentos internacionais de petróleo da China. 0 modelo de investimento incorpora nove fatores, como a disponibilidade de recursos de petróleo e de estabilidade política. Os fatores de ponderação para cada entrada foram determinados utilizando o AHP e lógica Fuzzy.

\section{- Artigos publicados em 2007}

Segundo Balasubramaniam, Boyle e Voulvoulis (2007), internacionalmente, a contaminação por petróleo é bastante difundida e apresenta sérios riscos ambientais, portanto, sua remediação é essencial. A implementação de opções de recuperação torna-se mais complexa com a influência de diferentes grupos de stakeholders nos resultados do processo decisório., A análise multicritério vem sendo utilizada nesse contexto devido à sua capacidade de incorporar as preferências de cada um dos interessados. A pesquisa investiga formas de melhorar o processo de ponderação. 0 estudo demonstra a utilidade do swing de pesos, determina o tipo de participantes e quantos podem ser incluídos no processo de decisão. 0 método ELECTRE 111 e a Soma Ponderada foram utilizados na avaliação de alternativas para remediação de contaminação por petróleo.

\section{- Artigos publicados em 2008}

Massara et al. (2008) analisaram a influência de vários parâmetros que determinam a expansão da rede de distribuição de gás natural em certas localidades através do uso do software Decision lens, um programa de decisão com base no AHP. Como estudo de caso, oito cidades da região administrativa de Araçatuba próximas do Gasoduto Bolívia-Brasil - Gasbol foram selecionadas e, como conclusão, foi identificado que os indicadores de desenvolvimento urbano e as atividades econômicas são determinantes para o consumo de energia e que devem contribuir para a expansão do gasoduto de gás natural.

De acordo com Sharma e Gandhi (2008), o petróleo entra em contato com o pessoal de operação e manutenção durante o manuseio, o armazenamento e a entrega. Os problemas de saúde decorrentes desse contato em longo prazo são graves. Pesquisadores identificaram os perigos e os problemas de saúde relacionados aos diferentes tipos de óleos lubrificantes em uso. No entanto, não é comum a aplicação de métodos matemáticos para avaliação (qualitativa/ quantitativa) da segurança no manuseio desses óleos. 0 principal objetivo do estudo é fazer essa avaliação. Para isto, os métodos AHP e de projeção do vetor são utilizados, considerando os atributos de segurança identificados (propriedades físicas e químicas que afetam o meio ambiente, o ser humano e o sistema) pelos pesquisadores. A metodologia proposta é útil para comparar diferentes alternativas de óleo lubrificante com uma alternativa ideal e ajuda a selecionar a opção mais segura entre todas as escolhas possíveis.

Xie et al. (2008) apresentaram um método para a seleção de um navio a partir de um grupo de candidatos que servirá como referência para novos desenhos de navio. 0 método é baseado no Evidential Reasoning e é dividido em três fases: identificação dos navios candidatos adequados, avaliação de atributos tecnoeconômicos convencionais, avaliação qualitativa e, finalmente, a agregação de todos os atributos usando a abordagem Evidential Reasoning. Um exemplo de seleção de navio petroleiro é utilizado e os resultados gerados pelo método incluem a classificação dos navios candidatos e indicaçaõ de seus pontos fortes e fracos.

\section{- Artigos publicados em 2009}

Araujo e Almeida (2009) apresentaram uma aplicação da metodologia multicritério na seleção de investimentos estratégicos em petróleo e gás no Nordeste do Brasil usando o método PROMETHEE 11. Uma aplicação numérica é apoiada por dados reais do planejamento estratégico de uma empresa de petróleo para o período 2008-2012.

Brito e Almeida (2009) desenvolveram um modelo para avaliação e classificação de riscos em tubulações para o bombeio e transporte de gás natural. 0 modelo utilizado baseia-se na aplicação de conceitos da MAUT à análise de árvores de riscos. A aplicação do modelo a uma situação fundamentou a sua validação.

Celik e Topcu (2009) utilizaram o método ANP (Analytic Network Process) para analisar e quantificar as responsabilidades ambientais (no ambiente maritimo) dos stakeholders em uma operação de exploração de petróleo. Segundo os autores, essa abordagem garante a justa penalização de cada stakeholder em uma situação de derramamento de óleo real.

Gomes, Rangel e Maranhão (2009) abordam o problema de selecionar a melhor alternativa para o destino das reservas de gás natural descobertas recentemente no campo de Mexilhão (Bacia de Santos, Brasil). 0 estudo tem o objetivo de: (1) criar um mecanismo que auxilie no processo de análise e seleção das melhores alternativas para o destino do gás natural; (2) permitir que os decisores escolham as 
opções de investimento mais bem alinhadas com as expectativas e objetivos estratégicos da empresa; e (3) permitir que os decisores identifiquem de forma mais sistemática, clara e abrangente novas oportunidades para atuar ou em que estão interessados em atuar. A análise multicritério empregada no estudo utiliza o método TODIM, método multicritério discreto fundamentado na Teoria dos Prospectos. A aplicação do método TODIM mostra que ele pode ser bastante útil na recomendação de opções de projetos no upstream devido ao fato de ele identificar claramente as alternativas mais importantes em face dos cenários testados e em relação aos critérios utilizados.

Jafari, Nejadi e Abiri (2009) desenvolveram um método de avaliação de risco que incorpora atributos qualitativos e quantitativos, o método AHP e questionários para simular os principais riscos na área de um campo de gás.

Montazer, Saremi e Ramezani (2009) afirmam que qualquer processo de decisão lida com duas questões principais: avaliação e classificação das alternativas com base em seu desempenho. A avaliação das alternativas depende, em grande parte, dos especialistas e de seus conhecimentos, podendo aumentar a incerteza no processo decisório. Na pesquisa, os autores discutem a arquitetura de um sistema Fuzzy de apoio à decisão. Esse sistema compreende dois módulos: um módulo de avaliação Fuzzy e um módulo Fuzzy de ordenação (que é uma versão difusa do método ELECTRE 111). Dessa forma, a ordenação final é resultado de um sistema que considera a incerteza existente nas avaliações. 0 sistema proposto foi aplicado a um caso real no processo de seleção de fornecedor em uma das maiores empresas do setor de petróleo iraniano, a OIEC (Oil Industries' Engineering \& Construction) e os resultados são discutidos.

Wang et al. (2009) introduziram o método AHP para avaliar exaustivamente um método baseado em GIS (Geographic Information Systems) de classificação e categorização de riscos em oleodutos e gasodutos

Yu, Tian e Xie (2009) utilizaram o método AHP para verificar falhas em dutos de aço (especificamente, gasodutos que abastecem cidades) causadas por corrosão e, também, para a identificação de riscos. Uma pesquisa foi realizada para identificar as fontes de risco e o método AHP serviu para geração de pesos para cada fonte de risco e para cada tipo de falha por corrosão.

\section{- Artigos publicados em 2010}

Amiri (2010) apresenta uma metodologia para avaliar alternativas de projetos e ajudar o decisor a selecionar o melhor investimento através de seis critérios de comparação entre as alternativas. 0 método AHP é utilizado para analisar a estrutura do problema de seleção de projetos e para determinar os pesos dos critérios e o método TOPSIS Fuzzy é usado para obter a ordenação final. A aplicação para ilustrar a utilização do modelo é feita na Companhia Nacional Iraniana de Petróleo.

Brito, de Almeida e Mota (2010) abordam um modelo multicritério de avaliação de riscos em dutos de gás natural e de classificação de seções de dutos em categorias de risco. 0 modelo integra a Teoria da Utilidade e o método ELECTRE TRI e visa auxiliar empresas na gestão de riscos e na tomada de decisão considerando as múltiplas dimensões de risco que podem surgir nos acidentes em dutos. A fim de verificar a eficácia do modelo estabelecido, uma aplicação numérica com base em um estudo de caso real é apresentada.

Dey (2010) desenvolveu uma metodologia para gerenciamento de riscos do projeto através da análise de risco em projeto. 0 autor desenvolve um quadro conceitual de gestão de risco usando AHP combinado com o mapa de risco. 0 modelo proposto foi aplicado a um projeto de construção do oleoduto de petróleo com cerca de $1.500 \mathrm{~km}$ na Índia. 0 autor afirma que o método AHP combinado com o mapa de risco é muito eficaz para gerenciamento de riscos.

Jozi e lraokhahi (2010) desenvolveram uma metodologia que utiliza o método AHP para avaliar os riscos ambientais nas linhas de gasodutos. Apresenta estudo de caso sobre as linhas de gasodutos de Tasooj-Salmas, no lrã.

Massara e Udaeta (2010) analisam a expansão da infraestrutura de distribuição de gás natural identificando as prioridades de uma grande metrópole, São Paulo, utilizando o método AHP. 0 modelo apresenta como um estudo de caso específico a integração entre o uso do gás natural e os bairros de São Paulo.

Ratnayake e Markeset (2010) propõem uma metodologia para avaliar se a medida do desempenho da integridade técnica (IT) está de acordo com o objetivo dos responsáveis pela GIT (gestão de integridade técnica). Também avaliam como as metas de SMS e os interesses financeiros são implementados no processo de seleção de uma estratégia ótima para manutenção em uma instalação de produção de P\&G, através de metodologia AHP. Para verificar sua viabilidade, foi realizado um estudo de caso em uma instalação localizada na plataforma continental norueguesa.

Shafiq e Silvianita (2010) utilizaram o método AHP para determinar o fator de risco para falhas de dutos, tanto na situação onshore quanto na situação offshore. 0 estudo de caso envolveu a detecção de falhas em duas redes de dutos, na Malásia e na Indonésia.

Song, Tang e Jiang (2010) desenvolveram uma metodologia que combina o método AHP (análise subjetiva) e o Entropy Weight Method (análise 
quantitativa), que escolhe o melhor método de detecção de enxofre no óleo cru. A metodologia é adotada pela refinaria Du Shanzi, na China.

\section{- Artigos publicados em 2011}

Dawotola, Van Gelder e Vrijling (2011) construíram um modelo para o gerenciamento de risco de dutos de óleo cru baseado no método AHP. 0 método AHP é utilizado para obter a probabilidade de fracasso relativo para os atributos de mecanismos de falha. Um estudo de caso de um oleoduto do mundo real é utilizado para demonstrar a aplicação do método proposto.

Tan et al. (2011) propuseram uma metodologia baseada em risco para a inspeção e manutenção em duas unidades de refinaria de óleo de Fujian, na China. 0 método AHP é utilizado para selecionar a estratégia mais viável para a manutenção dos equipamentos, que foram localizados em cada escala de classificação de risco identificado.

Wang et al. (2011) desenvolveram um sistema de gestão de risco baseada em GIS Web e o método AHP para melhorar a gestão da segurança de um oleoduto. Exemplos comparando o sistema proposto e os sistemas de gestão de risco tradicionais apontam que o primeiro é mais eficiente e mais fácil de aplicar.

Yang, Khan e Sadiq (2011) propuseram uma abordagem híbrida usando a lógica Fuzzy e o método AHP como uma ferramenta robusta para a priorização das questões ambientais nas operações de óleo e gás na situação offshore. Uma aplicação da abordagem proposta é demonstrada através de um exemplo numérico.

Yildirim e Yomralioglu (2011) sugerem um método que determina a rota ótima de um gasoduto utilizando o método AHP e o GIS. A eficácia desse método foi comprovada através de um estudo de caso.

\section{- Artigos publicados em 2012}

Zhang, Gao e Liu (2012) utilizam o método AHP e a lógica Fuzzy para propor um modelo de avaliação global de risco para os projetos de construção de poços aliviadores em campos de petróleo e gás. Um estudo de caso no mar da China Meridional é utilizado e os resultados da avaliação demonstram que a taxa de sucesso na perfuração desse poço de alívio é estimado em 48,7\%, proporcionando base informacional necessária para tomar decisões sobre como controlar os riscos existentes.

\section{Conclusão}

A pesquisa alcançou o seu objetivo principal: mapear as principais características das modelagens de AMD no âmbito da indústria de petróleo e gás. Foi apresentada uma pesquisa de revisão de literatura sobre o uso de métodos de auxílio multicritério à decisão no planejamento e gerenciamento do setor de petróleo e gás. Ao todo, 48 trabalhos selecionados da base Scopus ou da base ISI Web of Knowledge foram analisados. A análise desses artigos permitiu o mapeamento dessas publicações à luz dos seguintes descritores: método multicritério utilizado; evolução cronológica de publicação; tipo de fonte energética; segmento da cadeia produtiva; e tipologia da localidade das instalações.

Como resultado do estudo, os dois primeiros registros de documentos encontrados em uma das bases utilizadas na pesquisa e que contribuem para o tema proposto foram publicados no ano de 1996. Pode-se observar também que o maior número de trabalhos analisados foi publicado no ano de 2010 (8 artigos). É interessante notar que o método AHP é o mais utilizado, no contexto dos artigos analisados, para planejamento e gestão na indústria de $\mathrm{P} \& \mathrm{G}$, representando 60,94\% das ocorrências nesses artigos. Com referência aos descritores utilizados na pesquisa (fonte energética, segmento da cadeia produtiva e posicionamento geográfico), conclui-se que a maioria dos artigos analisados abordou petróleo $(41,67 \%)$, atividades midstream $(56,25 \%)$ e instalações onshore $(47,92 \%)$.

0 presente trabalho limitou-se a aplicar a metodologia à análise de periódicos indexados em duas bases de indexação disponíveis no portal de periódicos da Capes - observa-se que essas bases foram pesquisadas apenas com o filtro "artigos", ou seja, não se limitou a pesquisa por área de conhecimento, o que permitiu uma maior abrangência da pesquisa. De uma forma geral, conclui-se que a limitação maior desta pesquisa está associada ao fato da amostra contemplar apenas os artigos indexados no Scopus ou ISI Web of Science, apesar da qualificação dos indexadores destas bases, contribuições importantes, ainda não indexadas nas mesmas, podem não ter sido consideradas na pesquisa.

Com base no estudo desenvolvido, na elaboração da metodologia proposta e na experiência obtida no decorrer da sua aplicação são propostas as seguintes sugestões para desenvolvimentos futuros: pesquisar em outras bases como, por exemplo, a Scielo, a Engineering Village e a EBSCO.

Esta pesquisa também identifica que o uso de métodos multicritério é ainda pouco explorado em temas como: gás, downstream e offshore. Outra sugestão é a investigação sobre aplicações de métodos não compensatórios à solução de problemas da indústria de petróleo e gás.

\section{Referências}

AGÊNCIA NACIONAL DO PETRÓLEO, GÁS NATURAL E BlOCOMBUSTIVEIS - ANP. Glossário. Disponivel em: <www.anp.gov.br>. Acesso em: 16 nov. 2010. 
ALIDI, A. S. A multiobjective optimization model for the waste management of the petrochemical industry. Applied Mathematical Modelling, v. 20, n. 12, p. 925-933, 1996. http://dx.doi.org/10.1016/S0307-904X(96)00106-0

AMIRI, M. P. Project selection for oil-fields development by using the AHP and fuzzy TOPSIS methods. Expert Systems with Applications, v. 37, n. 9, p. 6218-6224, 2010. http:// dx.doi.org/10.1016/j.eswa.2010.02.103

ARAUJO, A. G.; ALMEIDA, A. T. Decision making in the selection of the investments in oil and gas: An application using the promethee method. Gestao \& Produção, v. 16, n. 4, p. 534-543, 2009.

BALASUBRAMANIAM, A.; BOYLE, A. R.; VOULVOULIS, N. Improving petroleum contaminated land remediation decision-making through the MCA weighting process. Chemosphere, v. 66, n. 5, p. 791-798, 2007. http:// dx.doi.org/10.1016/j.chemosphere.2006.06.039

BERTOLINI, M.; BEVILACQUA, M. A combined goal programming - AHP approach to maintenance selection problem. Reliability Engineering \& System Safety, v. 91, n. 7, p. 839-848, 2006. http://dx.doi.org/10.1016/j. ress.2005.08.006

BRITO, A. J.; DE ALMEIDA, A. T. Multi-attribute risk assessment for risk ranking of natural gas pipelines. Reliability Engineering \& System Safety, v. 94, n. 2, p. 187-198, 2009. http://dx.doi.org/10.1016/j. ress.2008.02.014

BRITO, A. J.; DE ALMEIDA, A. T; MOTA, C. M. M. A multicriteria model for risk sorting of natural gas pipelines based on ELECTRE TRI integrating utility theory. European Journal of Operational Research, v. 200, n. 3, p. 812-821, 2010. http://dx.doi.org/10.1016/j.ejor.2009.01.016

CELIK, M.; TOPCU, Y. 1. Use of an ANP to prioritize managerial responsibilities of maritime stakeholders in environmental incidents: An oil spill case. Transportation Research, v. 14, n. 7, p. 502-506, 2009.

CHEN, H. J. et al. Dual-factor method for development optimization of oil shale bearing fields. Geology in China, v. 38, n. 3, p. 742-749, 2011.

COSTA, H. G. Modelo para webibliomining: proposta e caso de aplicação. Revista da FAE, v. 13, n. 1, p. 115-126, 2010.

DAWOTOLA, A. W.; VAN GELDER, P. H. A. J. M.; VRIJLING, J. K. Decision analysis framework for risk management of crude oil pipeline system. Advances in Decision Sciences, 2011. http://dx.doi.org/10.1155/2011/456824

DEY, P. K. Integrated approach to project feasibility analysis: A case study. Impact Assessment and Project Appraisal, v. 19, n. 3, p. 235-245, 2001a. http://dx.doi. org/10.3152/147154601781766989

DEY, P. K. A risk-based model for inspection and maintenance of cross-country petroleum pipeline. Journal of Quality in Maintenance Engineering, v. 7, n. 1, p. 25-41, 2001b. http://dx.doi.org/10.1108/13552510110386874

DEY, P. K. Project risk management: A combined analytic hierarchy process and decision tree approach. Cost Engineering, v. 44, n. 3, p. 13-26, 2002.

DEY, P. K. Decision support system for inspection and maintenance: A case study of oil pipelines. IEEE Transactions on Engineering Management, v. 51, n. 1, p. 47-56, 2004. http://dx.doi.org/10.1109/ TEM.2003.822464

DEY, P. K. Integrated project evaluation and selection using multiple-attribute decision-making technique. International Journal of Production Economics, v. 103, n. 1, p. 90-103, 2006. http://dx.doi.org/10.1016/j. ijpe.2004.11.018
DEY, P. K. Managing project risk using combined analytic hierarchy process and risk map. Applied Soft Computing Journal, v. 10, n. 4, p. 990-1000, 2010. http://dx.doi. org/10.1016/j.asoc.2010.03.010

DEY, P. K.; GUPTA, S. S. Decision support system for pipeline route selection. Cost Engineering, v. 41, n. 10, p. 29-35, 1999.

DEY, P. K.; MUKHERJEE, S. K. Project risk management in analytic framework. International Journal of Industrial Engineering, v. 12, n. 4, p. 419-433, 2005.

DEY, P. K.; TABUCANON, M. T.; OGUNLANA, S. O. Petroleum pipeline construction planning: A conceptual framework. International Journal of Project Management, v. 14, n. 4, p. 231-240, 1996. http://dx.doi.org/10.1016/02637863(95)00092-5

DEY, P. K. Analytic hierarchy process analyzes risk of operating cross-country petroleum pipelines in india. Natural Hazards Review, v. 4, n. 4, p. 213-221, 2003. http:// dx.doi.org/10.1061/(ASCE)1527-6988(2003)4:4(213)

GOMES, L. F. A. M.; RANGEL, L. A. D.; MARANHÃO, F. J. C. Multicriteria analysis of natural gas destination in Brazil: An application of the TODIM method. Mathematical and Computer Modelling, v. 50, n. 1-2, p. 92-100, 2009. http://dx.doi.org/10.1016/j.mcm.2009.02.013

HO, W.; XU, X.; DEY, P. K. Multi-criteria decision making approaches for supplier evaluation and selection: A literature review. European Journal of Operational Research, v. 202, n. 1, p. 16-24, 2010. http://dx.doi. org/10.1016/j.ejor.2009.05.009

HUI Z.; DELl G. Screening the key techniques for oil \& gas drilling in consideration of safety. Natural Gas Industry, v. 25, n. 4, p. 77-78, 2005.

JAFARI, H. R.; NEJADI, A.; ABIRI, J. A. Risk assessment of industrial sites of Asalouye by using AHP technique and GIS. Journal of Environmental Studies, v. 35, n. 49, p. 53-60, 2009.

JIANG, H-Y. et al. Application of AHP on the choice of pipelines river-acrossing project. Journal of Southwestern Petroleum Institute, v. 27, n. 2, p. 80-83, 2005.

JOZI, S. A.; IRAOKHAHI, M. Environmental risk assessment of gas pipelines by using of AHP combined method. Journal of Environmental Studies, v. 36, n. 53, p. 107-120, 2010.

Ll, D.; ZHANG, C. P. The technical and economic synthetical estimating of Luodai natural gas field rebuilding. Wutan Huatan Jisuan Jishu, v. 27, n. 4, p. 351-353, 2005.

LIU, X. Models and method for vendor selection based on supply chain. Chinese Journal of Scientific Instrument, v. 26, p. 890-892, 2005.

MASSARA, V. M.; UDAETA, M. E. M. Multi-criteria evaluation of the expansion of natural gas distribution network by the urban dynamics. Journal of Urban and Environmental Engineering, v. 4, n. 2, p. 55-62, 2010. http://dx.doi. org/10.4090/juee.2010.v4n2.055062

MASSARA, V. M. et al. Natural gas pipeline expansion in the energy and urban planning using the analytic hierarchy process and the urban dynamics. Science and Engineering Journal, v. 17, p. 43-50, 2008.

MÉXAS, M. P.; QUELHAS, O. L. G.; COSTA, H. G. Prioritization of enterprise resource planning systems criteria: Focusing on construction industry. International Journal of Production Economics, v. 139, n. 1, p. 340-350, 2012. http://dx.doi.org/10.1016/j.ijpe.2012.05.025

MONTAZER, G. A.; SAREMI, H. Q.; RAMEZANI, M. Design a new mixed expert decision aiding system using fuzzy ELECTRE 111 method for vendor selection. Expert Systems with Applications, v. 36, n. 8, p. 10837-10847, 2009. http://dx.doi.org/10.1016/j.eswa.2009.01.019 
RATNAYAKE, R. M. C.; MARKESET, T. Methodology and theory: Technical integrity management: Measuring HSE awareness using AHP in selecting a maintenance strategy. Journal of Quality in Maintenance Engineering, v. 16, n. 1, p. 44-63, 2010. http://dx.doi. org/10.1108/13552511011030327

RODRIGUEZ, D. S. S.; COSTA, H. G.; DO CARMO, L. F. R. R. S. Métodos de auxílio multicritério à decisão aplicados a problemas de PCP: Mapeamento da produção em periódicos publicados no Brasil. Gestão \& Produção, v. 20 , n. 1, p. 134-146, 2013. http://dx.doi.org/10.1590/ S0104-530X2013000100010

SADIQ, R. et al. Risk-based decision-making for drilling waste discharges using a fuzzy synthetic evaluation technique. Ocean Engineering, v. 31, n. 16, p. 1929-1953, 2004.

SADIQ, R. et al. Evaluation of Generic Types of Drilling Fluid Using a Risk-Based Analytic Hierarchy Process. Environmental Management, v. 32, n. 6, p. 778-787, 2003. http://dx.doi.org/10.1007/s00267003-0009-2

SHAFIQ, N.; SILVIANITA. Prioritizing the pipeline maintenance approach using analytical hierarchical process. International Review of Mechanical Engineering, v. 4, n. 3 p. 346-352, 2010.

SHARMA, B. C.; GANDHI, O. P. Safety assessment of lubricating oil using AHP and vector projection method. Industrial Lubrification and Tribology, v. 60, n. 5, p. 259-265, 2008. http://dx.doi.org/10.1108/00368790810895204

SII, H. S.; WANG, J. A design-decision support framework for evaluation of design options/proposals using a composite structure methodology based on the approximate reasoning approach and the evidential reasoning method. Proceedings of the Institution of Mechanical Engineers, v. 217, n. 1, p. 59-76, 2003. http://dx.doi. org/10.1243/09544080360562990

SONG, Z. Z.; TANG, F.; JIANG, Q. Z. Technical, economic and environmental comprehensive evaluation on sulfurcontaining crude processing scheme. Modern Chemical Industry, v. 30, n. 8, p. 83-86, 2010.

TAN, Z. et al. W. An evaluation of maintenance strategy using risk based inspection. Safety Science, v. 49, n. 6, p. 852-860, 2011.

TESFAMARIAM, S.; SADIQ, R. Risk-based environmental decision-making using fuzzy analytic hierarchy process
(F-AHP). Stochastic Environmental Research and Risk Assessment, v. 21, n. 1, p. 35-50, 2006. http://dx.doi. org/10.1007/s00477-006-0042-9

WANG, W. Q. et al. Research on the risk grading division of oil and gas pipeline based on WebGiS. Journal of Petrochemical Universities, v. 22, n. 4 p. 63-66, 2009.

WANG, W. Q. et al. Risk management and emergency response of oil pipeline based on web GIS. Journal of Petrochemical Universities, v. 24, n. 5, p. 77-82, 2011.

$\mathrm{XIAO}, \mathrm{W}$. et al. Multiobjective linear programming model on injection oilfield recovery system. Computers and Mathematics with Applications, v. 36, n. 5, p. 127-135, 1998.

$\mathrm{XIE}, \mathrm{X}$. et al. Ship selection using a multiple-criteria synthesis approach. Journal of Marine Science and Technology, v. 13, n. 1, p. 50-62, 2008. http://dx.doi.org/10.1007/ s00773-007-0259-4

WU, H.; BARNES, D. A literature review of decision-making models and approaches for partner selection in agile supply chains. Journal of Purchasing and Supply Management, v. 17, n. 4, p. 256-274, 2011. http:// dx.doi.org/10.1016/j.pursup.2011.09.002

YANG, M.; KHAN, F. 1.; SADIQ, R. Prioritization of environmental issues in offshore oil and gas operations: A hybrid approach using fuzzy inference system and fuzzy analytic hierarchy process. Process Safety and Environmental Protection, v. 89, n. 1, p. 22-34, 2011. http://dx.doi.org/10.1016/j.psep.2010.08.006

YANG, X. et al. Fuzzy math evaluation method for China's international oil investments. Journal of Tsinghua University, v. 46, n. 6, p. 855-857, 2006.

YILDIRIM, V.; YOMRALIOGLU, T. NABUCCO pipeline route selection through Turkey comparison of a GIS-based approach to a traditional route selection approach. Oil Gas European Magazine, v. 37, n. 1, p. 20-24, 2011.

YU, J. C.; TIAN, J. X.; XIE, D. L. Hazard resources and their weightings in risk assessment system of the steel gas pipeline corrosion. Journal of Northeastern University, v. 30, p. 23-25, 2009.

ZHANG, H.; GAO, D.; LIU, W. Risk Assessment for Liwan Relief Well in South China Sea. Engineering Failure Analysis, v. 23, p. 63-68, 2012. http://dx.doi.org/10.1016/j. engfailanal.2012.02.005

\title{
The adoption of Multicriteria Decision Aid (MCDA) methods in the management and planning of the petroleum and gas industry: a bibliographical study
}

\begin{abstract}
The application of multicriteria methods in the planning and management of the oil and gas industry has been studied by various authors. However, these studies are distributed in a scattered manner in the literature, which creates difficulties in obtaining an overview. To provide a systematic view on this matter, this paper presents a bibliographical mapping of papers that approach the use of multicriteria methods for planning and management in the oil and gas industry. The reviewed documents were selected through a search in the Scopus and ISI (Web of Knowledge) databases. Among the study results, it is found that the most commonly used multicriteria method was AHP (Analytic Hierarchy Process). Furthermore, it is observed that the majority of multicriteria method applications are related to oil, midstream activities and onshore facilities.
\end{abstract}

Keywords

MCDA. Planning. Management. Oil. Gas. 